\title{
Reciclaje tecnológico al SERVICIO de la ciencia
}

\author{
Lorena Cardona ${ }^{1}$, \\ Paula A. Ortiz ${ }^{2}$, \\ AlejandRo Restrepo ${ }^{3}$
}

\section{Resumen:}

En este trabajo se hace una revisión de los desarrollos científicos logrados a partir del reciclaje tecnológico de partes de computador, más específicamente, de unidades de CD/DVD y ratones. Estos elementos, que con frecuencia son desechados generando serios problemas de contaminación ambiental, son en sí mismos elementos sofisticados y de gran precisión, que al ser producidos en masa pueden adquirirse a muy bajo costo. Estas características los convierten en equipos interesantes para trabajos de investigación científica que buscan alternativas simples, de bajo costo y miniaturización de equipos (laboratorio en un chip). Microscopios, interferómetros, escáneres, odómetros, manómetros, péndulos, codificadores, entre otros, son los desarrollos que se han logrado a partir de la reutilización de estos elementos. Aquí se muestra el principio de funcionamiento de estas partes de computador, las aplicaciones a que han sido objeto desde su invención y otros campos de aplicación potenciales como conclusión de la revisión realizada.

\section{Palabras clave:} costo.

Reciclaje tecnológico, ratón, CD/DVD, laboratorio en un chip, bajo

1 Ingeniera mecánica, $\mathrm{MsC}$ en automática, docente investigadora en el Instituto Tecnológico Metropolitano, lorenacardona@itm.edu.co

2 Ingeniera de control, $\mathrm{MsC}_{\mathrm{s}}$ en automática, docente auxiliar en el Instituto Tecnológico Metropolitano, paulaortiz@itm.edu.co

3 Ingeniero mecánico, $\mathrm{PhD}$ en sistemas, docente investigador en el Instituto Tecnológico Metropolitano, alejandrorestrepo@itm.edu.co 


\section{Abstract:}

This paper is a review of scientific developments achieved from recycled parts of computer technology, more specifically, CD/DVD drives and mice. These elements, which are often discarded causing serious environmental pollution problems, are sophisticated high precision components, and, as they are produced in mass, can be acquired at very low cost. These features make them interesting for scientific research in seeking simplicity, low cost, and miniaturization of equipment (lab on a chip). Microscopes, interferometers, scanners, odometer, manometers, pendulums, encoders, among others, are the applications that have been achieved from the reuse of these elements. Here we present the working principle of these computer parts, the applications they have had since their invention and the prospective application fields as the conclusion of this revision work.

\section{Keywords:}

Technology recycling, mouse, CD/DVD, lab on a chip, low cost. 


\section{INTRODUCCIÓN}

Debido a las rápidas mejoras en las tecnologías de manufactura electrónica, los computadores se han convertido en productos electrónicos de corta vida. Este fenómeno resulta en una gran cantidad de computadores desechados, los cuales contienen materiales peligrosos como plomo, mercurio y bario, que pueden contaminar seriamente el medio ambiente (Lee et al., 2004). Cerca de 500 millones de computadores alcanzaron el fin de su vida útil entre 1994 y 2004 en el mundo, y se estima que alrededor de 1 billón de computadoras serán desechadas para el año 2010 (Lee et al., 2004a). En el caso específico de Colombia, se estima que se podrían acumular entre 13 y 22 millones de computadores desechados para el año 2013, si no se avanza en su gestión y aprovechamiento (Ott, 2008).

Una solución para este problema está en el reciclaje de los materiales que conforman dichos computadores, extrayendo la materia prima de sus elementos, sin embargo, el costo de desmantelar y reciclar los materiales de un computador es tan alto que muchos países optan por enviar los desechos a países en desarrollo (Puckett \& Smith, 2002), razón por la cual ciudades como Giuyu (China) están sepultadas bajo montañas de computadoras en desuso. Colombia no tiene actualmente ninguna legislación sobre el tratamiento de desechos electrónicos ni tampoco suficiente infraestructura para reciclar este tipo de residuos (Ott, 2008).

Por esto es importante pensar no solo en el reciclaje de computadores si no también en modelos de reutilización y reacondicionamiento. Una entidad que se destaca en esta área es Computadores para Educar (Marthaler, 2008), la cual reacondiciona computadores donados y los regala a instituciones educativas públicas. Adicionalmente, desde el año 2007 se han diseñado Kits de Robótica compuestos de varios residuos que quedan del proceso de reacondicionamiento (fuentes de potencia, interruptores y pequeños motores eléctricos), los cuales se entregan a instituciones beneficiarias del programa (Marthaler, 2008). 
Siguiendo esta misma tendencia, aunque no necesariamente inspirados en el problema de contaminación ambiental expuesto, muchos investigadores han desarrollado trabajos en los que diferentes partes de computador se reutilizan y adaptan para dar soluciones innovadoras y de bajo costo a problemas en ámbitos industriales, científicos o educativos, mientras se genera un impacto ambiental positivo. De este modo, se vislumbra una nueva solución para el problema de contaminación generado por los computadores: un reaprovechamiento de la tecnología presente en las diferentes partes de estos equipos que puede servir sus propósitos en otras áreas. En este artículo se presentan los resultados de investigaciones que han permitido reutilizar algunas partes de computador en diferentes campos, aprovechando la tecnología que hay en ellos. El trabajo se centra en dos elementos, a saber, el ratón óptico y la unidad de CD/DVD. Inicialmente se explica el funcionamiento de estos dos dispositivos, luego se presentan las aplicaciones alternativas que han tenido ambos elementos y finalmente se presentan las conclusiones, exponiendo las tendencias y vacíos encontrados en la aplicación de los ratones de computador y las unidades de CD/DVD.

\section{Funcionamiento de los dispositivos}

Aquí se describe el funcionamiento del ratón de computador y de la unidad de CD/DVD, que son objeto de estudio en este artículo.

\subsection{Ratón de Computador}

En la actualidad se encuentran dos tipos de ratón, el optomecánico (de bola) y el óptico, aunque el ratón opto-mecánico se emplea cada vez menos. A continuación se describe el funcionamiento de cada uno de ellos.

\subsubsection{Ratón Opto-Mecánico}

Una bola dentro del ratón permanece en contacto con la superficie y rueda cuando el ratón es arrastrado. Dos rodillos dentro 
del ratón tocan la bola, uno de los cuales detecta el movimiento en la dirección $x$ y el otro detecta el movimiento en la dirección $y$. Cada uno de los rodillos está conectado a un eje con un disco ranurado. A un lado del disco hay un LED infrarrojo y al lado opuesto hay un sensor infrarrojo. Las ranuras en el disco rompen el haz de luz proveniente del LED de tal forma que el sensor infrarrojo lee pulsos de luz, cuya velocidad es directamente proporcional a la distancia recorrida. Un procesador convierte los pulsos en datos binarios que son llevados al computador, donde los drivers convierten el movimiento del rodillo en un movimiento del cursor en pixeles (Mei, 2005).

\subsubsection{Ratón Óptico}

En el ratón óptico, un diodo emisor de luz (LED) ilumina la superficie bajo el ratón. Una lente recoge la luz reflejada por la superficie y forma una imagen en un sensor. El sensor toma fotografías continuamente a medida que el ratón se desplaza lo suficientemente rápido (1500 cuadros por segundo o más rápido) como para que se superpongan imágenes secuenciales. Las imágenes se envían al motor de navegación óptica quien identifica características en las imágenes y sigue la pista de su movimiento. Esto se traduce en coordenadas $x$ e $y$ de movimiento del ratón (Agilent Technologies, 2001). Los nuevos ratones ópticos desarrollados no utilizan un LED si no un láser para iluminar la superficie, logrando mayor precisión.

\subsection{Unidad de CD/DVD}

Una unidad de CD/DVD, es un dispositivo capaz de leer la información contenida en un disco compacto (CD) y en un disco versátil digital (DVD). Los elementos básicos de una unidad de CD son: un cabezal óptico, un motor que hace girar el disco compacto y un motor que mueve el cabezal radialmente. En el CD o DVD, la información está escrita sobre una espiral, así, a medida que el disco gira, el cabezal óptico se desplaza radialmente para leer dicha información. La forma en que se leen los datos es la siguiente: 
un láser cuyo haz es colimado por unas lentes, es dirigido hacia una lente objetivo a través de un divisor de haz. La lente objetivo enfoca el haz en el disco y recoge la luz reflejada. Esta luz reflejada se dirige (en el divisor de haz) hacia los detectores, que producen una señal de lectura de datos, así como señales para auto-enfoque y seguimiento de pista (Mansuripur \& Sincerbox, 1997).

Una de las características más interesantes y de mayor aplicación de la unidad de CD/DVD es el auto-enfoque de la lente objetivo. La luz reflejada por el disco y recogida por la lente objetivo es llevada a un detector de cuadrantes a través de unas lentes astigmáticas. Cuando el sistema está enfocado, el detector recibe la misma cantidad de luz en sus cuatro cuadrantes. Cuando el disco está fuera de foco, el astigmatismo crea un punto alargado en el detector, proporcionando una señal de error de enfoque, que alimenta al actuador de bobina de voz que desplaza la lente objetivo para mantener el enfoque automático (Mansuripur \& Sincerbox, 1997).

\section{Aplicaciones del ratón de computador}

\subsection{Aplicaciones en Física}

La primera aplicación alternativa que se dio al ratón de computador fue llevada a cabo por Handler et al. (1996) quienes propusieron varios experimentos que podían llevarse a cabo con el uso de un ratón, Uno de los experimentos buscaba estudiar el movimiento armónico simple, incluyendo en su montaje los componentes de masa-resorte habituales, pero transfiriendo el movimiento de la masa a uno de los rodillos de un ratón opto-mecánico por medio de una cuerda. En un segundo experimento, si la constante del resorte se conocía, el dispositivo podía usarse para medir fuerzas, utilizando pesos de magnitud incierta y midiendo el movimiento del resorte. Un tercer experimento consistía en usar el dispositivo como transductor (conversor eléctrico-mecánico), convirtiendo datos de voltaje o temperatura en desplazamientos del resorte. 
Dos años después, Ochoa et al. (1998) idearon una aplicación para demostrar la ley de Lenz (el sentido de una corriente inducida es tal que su flujo se opone a la causa que la produce) que consistía en lanzar un imán a través de un tubo cilíndrico de cobre o aluminio y medir la posición del imán en función del tiempo, acoplando el imán a uno de los rodillos del ratón opto-mecánico por medio de una cuerda mientras un software leía la información del ratón.

$\mathrm{Ng} \&$ Ang (2004) investigaron la eficacia del ratón óptico para medir desplazamientos vibratorios ubicando el ratón cerca de la superficie de una pieza de metal conectada a un agitador. Determinaron que el ratón óptico tenía un alcance limitado de baja frecuencia $(5 \mathrm{~Hz})$ y baja amplitud $(0,2 \mathrm{~mm})$. Más adelante, $\mathrm{Ng} \&$ Ang (2005) utilizaron un ratón óptico para registrar la posición de un oscilador mecánico simple, utilizando una masa que colgaba de una cuerda unida a un resorte. Un pedazo de cartón, que servía como superficie de referencia, se pegaba a la cuerda de modo que un ratón óptico medía su desplazamiento. Luego, $\mathrm{Ng}$ et al. (2007) utilizaron un ratón óptico para lograr lecturas digitales y adquisición automática de datos en un manómetro de presión. En su trabajo, utilizaron un manómetro de tubo en U, colocando un flotador en uno de los brazos del tubo de tal manera que se moviera con el nivel de líquido y situaron un ratón óptico de tal forma que detectara el movimiento del flotador.

Gintautas \& Hübler (2009) diseñaron un péndulo haciendo un agujero en uno de los rodillos de un ratón opto-mecánico y encajando en este agujero una barra que funcionaba como péndulo. El ratón se conectaba al computador durante los experimentos, donde se convertía el movimiento del cursor en unidades de desplazamiento angular del péndulo, logrando una resolución de 0,0324 radianes.

\subsection{Aplicaciones en Robótica}

Desde el año 2003 los ratones ópticos han sido utilizados para medir la posición, orientación y/o velocidad de robots móviles. Para medir estas mismas variables, durante años se han usado codificadores acoplados a los ejes de los motores de accionamiento 
de los robots. Sin embargo, este sistema no funciona correctamente en superficies resbaladizas, puesto que cuando hay deslizamiento de las ruedas, el codificador no detecta el movimiento. Con el uso del ratón óptico se resuelve este problema gracias a que éste detecta directamente el movimiento de la superficie. Para medir el desplazamiento de un robot utilizando un ratón óptico, éste se dispone en la cara inferior del robot, mirando hacia el piso, de manera que al desplazarse el robot, el sensor detecte el desplazamiento sobre el suelo. Pero, aunque un ratón da una medida precisa del desplazamiento, no puede medir rotaciones, necesitándose para esto al menos dos ratones (Sorensen et al., 2003). Ng (2003) investigó la capacidad de un ratón óptico para funcionar como sensor de desplazamiento en dos dimensiones y concluyó que el ratón sólo podía obtener mediciones sobre objetos opacos y siempre que la distancia entre el objeto y el ratón fuera menor a $1,25 \mathrm{~mm}$.

En 2004 Cooney et al. (2004) y Bonarini et al. (2004) utilizaron dos ratones ópticos acoplados a la parte delantera y trasera de un robot para medir el desplazamiento de tres grados de libertad del robot. Si bien, utilizando sensores ópticos lograron una estimación fiable, como el ratón está diseñado para ser colocado muy cerca de la superficie ( $\mathrm{Ng}, 2003)$, en terrenos irregulares las medidas no son confiables. Por esta razón Lee et al. (2004a) utilizaron ratones ópticos en la misma forma utilizada por Cooney et al. (2004) pero combinaron esta información con las medidas de codificadores acoplados a las ruedas de tracción del robot, usando un filtro extendido de Kalman. Sin embargo, ante la presencia de deslizamientos, el filtro no respondió bien porque su funcionamiento se basaba en un modelo cinemático.

Diferentes trabajos se siguieron haciendo en torno a la estimación del desplazamiento de un robot móvil utilizando dos sensores de ratón óptico, tales como el trabajo de Lee \& Song (2004b) y Bonarini et al. (2005). Estos últimos presentaron además un procedimiento para reducir los errores no sistemáticos del sensor. Con este mismo objetivo se comenzaron a desarrollar otras 
alternativas que usaban más de dos ratones o que adicionaban otro tipo de sensores. Sekimori \& Miyazaki (2005) propusieron un método que utilizaba un filtro de Kalman para combinar la información de cuatro sensores de ratón óptico y una cámara global. Lograron buenas estimaciones de desplazamiento pero los errores en la estimación de la orientación tendían a aumentar con el tiempo. Kim \& Kim (2006) presentaron un método que utilizaba tres ratones ópticos dispuestos en forma de triángulo regular y propusieron un algoritmo de localización basado en el método de mínimos cuadrados, pero seguían presentándose problemas por variaciones de altura en el sensor.

Dado que se seguían buscando soluciones para el empleo de ratones ópticos en la estimación del movimiento de robots móviles, Minoni \& Signorini (2005) y Palacin et al. (2006) investigaron los factores que afectaban la precisión de los ratones ópticos para medir el desplazamiento de robots móviles. Encontraron que la linealidad del ratón óptico era muy buena con un coeficiente de $\mathrm{R}^{2}=0,9998$ (Palacin et al., 2006), pero que la calibración del sensor dependía fuertemente de la textura de la superficie (Minoni \& Signorini, 2005). Concluyeron que el ratón era sensible a la orientación y obtuvieron mejoras utilizando un arreglo de múltiples sensores con diferentes orientaciones (Minoni \& Signorini, 2005). Encontraron también que una pequeña variación de la distancia entre el sensor y la superficie tenía gran impacto sobre la precisión y que en suelos blandos el desplazamiento del ratón podía cambiar la superficie y confundir al algoritmo de procesamiento de imágenes (Palacin et al., 2006).

Por otra parte, descubrieron que el ratón tenía problemas para medir desplazamientos combinados (trayectorias diagonales y curvas) y que tenía un límite máximo de velocidad (Minoni \& Signorini, 2005). Más adelante, Jackson et al. (2007) realizaron un análisis comparativo de varias tecnologías de seguimiento, a saber, variedades de GPS, acelerómetros, telémetros láser, y los sensores del ratón óptico, y concluyeron que el ratón óptico era la única tecnología con precisión en el rango de milímetros siendo 
además la menos costosa, pero tenía como limitante la velocidad, pues solo daba lecturas confiables con velocidades menores a 7 $\mathrm{km} / \mathrm{h}$.

Teniendo en cuenta que una de las principales limitaciones del ratón óptico es que éste debe permanecer muy cerca al suelo y trabajar a baja velocidad, Bradshaw et al. (2007) trabajaron en el desarrollo de un sistema de medición añadiendo la lente de una cámara CCD para ampliar el espacio físico proyectado sobre el sensor. Así, aumentaron la distancia que el sensor podía viajar teniendo todavía correlación entre dos imágenes sucesivas para estimar el movimiento, superando las limitaciones de velocidad y logrando que el sistema pudiera ser colocado a una distancia razonable de la superficie. Buscando mejoras en la precisión, $\mathrm{Hu}$ et al. (2007) utilizaron dos sensores de ratón láser y propusieron un algoritmo para evadir obstáculos que comparaba la trayectoria medida por los sensores ópticos con una trayectoria de referencia. Una condición de choque se daba cuando la velocidad real se desviaba de la de referencia tanto en dirección como en magnitud. Kim \& Lee (2007) presentaron el mismo arreglo de tres sensores que usaron un año antes (Kim et al., 2006), pero esta vez para estimar la velocidad y no el desplazamiento de un robot móvil. Más adelante, Kim, \& Lee (2008) presentaron un método para calibrar la posición del arreglo poligonal de ratones debido a que una instalación de los ratones desviada de un polígono regular deterioraba la estimación de velocidad.

Posteriormente, Tunwattana et al. (2008) investigaron algunas características del ratón óptico para medir desplazamientos y concluyeron que con iluminación en ambos ejes del ratón, las lecturas en desplazamientos combinados mejoraban mucho y también que la sensibilidad del ratón era mayor en condiciones de aceleración que en condiciones de desaceleración. Hu et al. (2009) propusieron un método de calibración para eliminar los errores sistemáticos usando el principio de sensor de fusión, en el que las lecturas de todos los sensores deben reflejar el hecho de que están montados sobre un cuerpo rígido. 
Finalmente, el trabajo más reciente es el de Hyun et al. (2009), quienes diseñaron un sensor diferencial en el que se disponen dos ratones ópticos perpendiculares entre sí a diferentes distancias de un punto de cruz. Un divisor de haz en el punto de cruz da a los dos sensores una imagen de la misma área, pero el tamaño de la superficie proyectada difiere en ambos. Debido a que la diferencia de altura entre los dos sensores es constante, la distancia que el ratón se desplaza se puede calcular independientemente de las variaciones en la altura del robot.

\subsection{Otras Aplicaciones}

Aunque la medida del desplazamiento y velocidad de un robot móvil es la aplicación más estudiada para los ratones de computador, éste también ha tenido atención en otras áreas de la ciencia. Nakao et al. (1998) desarrollaron una técnica para introducir imágenes de documentos o fotografías al computador que consistía en acoplar un ratón óptico a una cámara y mover el ratón sobre el documento, tomando imágenes parciales que luego se fusionaban en una sola imagen. Yang \& Maeda (2000) presentaron un sistema para medir micro-caudales en líquidos que consistía en medir el tiempo que tardaba una burbuja de gas en atravesar un tubo de vidrio dispuesto entre el diodo emisor de luz (LED) y el fotodiodo (PD) de un ratón.

Cuando el tubo estaba lleno de aire, la luz de LED se dispersaba, y el PD se encontraba en OFF y cuando el tubo de vidrio estaba lleno de agua, enfocaba la luz del LED y el PD se encontraba en $\mathrm{ON}$. Al medir el intervalo de tiempo entre dos pares ON-OFF, se podía calcular el caudal. Ng (2004) utilizó un ratón óptico para medir la deformación viscoelástica de una lámina de polímero dispuesta verticalmente, cuyo extremo superior se fijaba a un soporte mientras que en su extremo inferior se colgaba un objeto de masa conocida. Unida a la lámina de polímero se disponía una superficie de referencia que se desplazaba a medida que la lámina se alongaba y su desplazamiento era medido por un ratón óptico. $\mathrm{Ng}$ \& Cheong (2004) utilizaron un ratón óptico para grabar posiciones 
de interés en un microscopio óptico equipado con una plataforma de desplazamiento $x y$. El ratón óptico se ubicaba boca arriba en la plataforma del microscopio y una superficie de referencia se fijaba al porta-objetos del microscopio, de tal forma que cuando la superficie se desplazaba, el ratón medía su movimiento y lo transmitía al computador. Donatell et al. (2005) emplearon dos ratones ópticos en un dispositivo para medir la posición lumbar en pacientes durante rutinas de ejercicio.

El ratón de computador también se ha utilizado para medir el movimiento de insectos sometidos a diferentes estímulos sensoriales. Esta aplicación fue desarrollada originalmente por Doherty \& Pires (1987) quienes reemplazaron la bola del ratón por una esfera pequeña y lo suficientemente ligera como para ser movida por el movimiento de un grillo de campo. Una versión actualizada se desarrolló más tarde empleando un ratón óptico para medir el movimiento de un animal mucho más pequeño y ligero, el parasitoide Ormia Ochracea (Mason et al., 2001). Sin embargo, esta versión logró una tasa de muestreo de tan sólo $40 \mathrm{~Hz}$, no pudiendo aplicarse para mediciones en línea de alta velocidad. Más tarde, Lott et al. (2007) desarrollaron una versión mejorada reemplazando la cámara dentro del ratón por otra con mayor frecuencia de muestreo $(2300 \mathrm{~Hz})$.

Wang et al. (2008) utilizaron el sensor de un ratón óptico como detector de formas de superficies y demostraron que el sensor permitía distinguir las formas y detectar la variación de altura en las superficies. La variación de la altura se calculó con base en el hecho de que la resolución del ratón cambiaba al variar la altura. Wang \& Shida (2009) propusieron un dispositivo para analizar la forma de una superficie que constaba de un bloque de madera de baja inercia en el cual se acoplaba la punta de un bolígrafo usada como sonda, manteniendo un contacto entre la sonda y la superficie a analizar por medio de un resorte ubicado entre la parte superior del bloque de madera y una carcasa. Cuando el dispositivo barría una superficie, un ratón óptico fijo medía el desplazamiento del bloque de madera en respuesta a las variaciones de altura de la superficie del objeto. 
Courreges et al. (2009) utilizaron un ratón óptico para controlar de manera remota la orientación de un brazo robótico usado en telesonografía. La orientación de la sonda podía definirse a partir de las coordenadas de uno de sus extremos, las cuales se controlaban con el movimiento del ratón óptico.

Tresanchez et al. (2009a) diseñaron un detector de monedas falsas que utiliza un ratón óptico para obtener imágenes parciales de la moneda que se estaba analizando, las cuales se comparaban con imágenes de referencia para la moneda correspondiente. Luego, los mismos investigadores (Tresanchez et al., 2009b) construyeron un codificador rotatorio incremental ubicando el sensor de un ratón óptico a una distancia fija de la superficie interna de una rueda rotatoria blanca, en la cual se dibujaba una línea de referencia en color negro. Como en un ratón se mantiene un nivel de iluminación promedio sobre la superficie por medio de un lazo de control, sobre la marca negra se producían cambios en el lazo de control para ajustar la iluminación. La detección de esta marca de referencia permitía contar el número de revoluciones, corregir errores acumulados y calibrar el número de conteos por la revolución (CPR). Sin embargo, Tresanchez et al. (2010) se dieron cuenta de que después de que se ponía en marcha el sistema, no se conocía la posición absoluta del codificador, limitando su uso como codificador relativo. Por esta razón, propusieron usar la capacidad de adquisición de imágenes del ratón óptico para leer un código binario impreso en la superficie interior de la rueda rotatoria midiendo la posición absoluta del codificador en tiempo real.

\section{Aplicaciones de la unidad DE CD/DVD}

En los últimos años ha habido un interés considerable por el uso de la tecnología de disco compacto para el desarrollo de sensores biológicos, micro-fluídica y la miniaturización de pruebas de diagnóstico rápido. Estos métodos toman ventaja de la escala nanomicroscópica de los discos ópticos comerciales, que los enmarca dentro de la tendencia actual de tecnologías de laboratorio-en-un- 
chip (LOC). Un LOC es un dispositivo que integra una o varias funciones de laboratorio en un chip único con un tamaño de sólo unos milímetros cuadrados (Kricka, 1998).

\subsection{Aplicaciones en Perfillometría}

Todos los trabajos desarrollados en perfilometría óptica que hacen uso de la unidad de CD/DVD se basan en el método de auto-enfoque: cuando se escanea una superficie con el láser de la unidad, un actuador que controla el desplazamiento de la lente objetivo mantiene el láser enfocado sobre la superficie. La posición de la lente objetivo es entonces una medida de la topografía local de la superficie.

El primer trabajo realizado en este sentido es el de Zhang \& Cai (1997a) quienes usaron el cabezal óptico de una unida de CD y un traductor piezoeléctrico (PZT) para medir el perfil de una superficie. El sistema de medición consistía en una cabeza óptica y dos plataformas impulsadas por motores piezoeléctricos. Un motor piezoeléctrico se utilizaba para impulsar la lente objetivo en el sistema de auto-enfoque y los otros motores se utilizaban para explorar el perfil de la superficie en sentido transversal. Más adelante, Zhang \& Cai (1997b) construyeron otro perfilómetro realizando dos cambios sobre el trabajo anterior. En vez de impulsar la lente objetivo para controlar el enfoque automático, fijaron la lente objetivo en el cabezal óptico y controlaron el movimiento del cabezal óptico en conjunto con un PZT, con el fin de mejorar la medición y la exactitud de la posición.

También usaron un interferómetro de Reigshaw para medir el movimiento del PZT. Ehrmann et al. (1998) diseñaron y construyeron un perfilómetro óptico donde el sensor estaba montado sobre una plataforma motorizada en $x$ e $y$. La medición de objetos en 3D se conseguía mediante el escaneo de una sección plana completa y luego moviendo o rotando el objeto para explorarlo en un plano diferente. De los datos de superficie almacenados se reconstruía un modelo de volumen del objeto. Bartoli et al. (2001a; 2001b) desarrollaron un perfilómetro en el cual el escaneo de la muestra, 
que se hacía en una sola dimensión, lo realizaba el motor de desplazamiento del cabezal óptico. Fan et al. (2001) desarrollaron un perfilómetro utilizando lectores ópticos de DVD, logrando mayor precisión que con los perfilómetros basados en lectores de CD. Islam et al. (2007) utilizaron el lector óptico de una unidad de DVD para medir la información del perfil y un ratón óptico para medir la posición del sensor. El uso del ratón hizo que el sistema fuera de bajo costo, a diferencia de las otras propuestas que usaban plataformas piezo-eléctricas.

\subsection{Aplicaciones en microscopía}

Benschop \& Rosmalen (1991) construyeron un microscopio óptico de barrido (SOM) usando un reproductor de $\mathrm{CD}$. El bajo peso y la construcción compacta permitieron desplazar el microscopio entero con respecto a un objeto estacionario. Excepto por el trabajo de Benschop et al., la mayoría de aplicaciones de la unidad de CD/ DVD en microscopía, están relacionadas con la microscopía de fuerza atómica (AFM). En AFM, las fuerzas interatómicas entre una sonda y la superficie inducen desplazamientos verticales en un voladizo microfabricado. Si el láser del cabezal de la unidad de CD/DVD se enfoca sobre el voladizo a través de la lente objetivo, el desplazamiento del motor que controla la posición de la lente para asegurar el enfoque, es proporcional al desplazamiento del voladizo.

Quercioli et al. (1999b) probaron este sistema para medir el desplazamiento vertical de un voladizo en AFM y los resultados demostraron que el sistema de detección era sensible y fiable. Hwu et al. (2006) utilizaron el mismo sistema modificando un microscopio comercial mediante la sustitución de su módulo de deflexión de haz por la cabeza de un lector óptico de CD/DVD. Hwu et al. (2009) presentaron el desarrollo de un módulo híbrido que combinaba un modo de barrido y un modo óptico (perfilometría óptica), en una sola cabeza de sensor. En el modo de barrido, el láser enfocado medía la posición de la sonda. En el modo de perfilometría óptica, la superficie de muestra se medía directamente con el haz de láser enfocado. El módulo podía ser utilizado en un microscopio 
óptico modificado o podía ser implementado como un sistema independiente.

\subsection{Aplicaciones en biología}

Jones (2005) desarrolló un biosensor óptico basado en la realización de una tinción de Gram directa sobre las células inmovilizadas en la superficie de datos de un CD. Las células teñidas en la superficie del CD causaban interferencia con la trayectoria de la luz de láser en el lector de la unidad de CD. Diferentes bacterias causaban efectos repetibles de atenuación de la luz que podían ser medidos usando sumas de comprobación y comparaciones a nivel de bits. Tibbe et al. (2002) desarrollaron un sistema de análisis celular para enumerar y diferenciar células alineadas magnéticamente. La unidad de CD permitía enfocar un haz de luz sobre las células alineadas manteniendo una iluminación óptima. La luz de fluorescencia emitida por las células se capturaba por la lente objetivo de la unidad de CD y luego era llevada a un detector de fluorescencia. Kim et al. (2005) utilizaron la capacidad de enfoque y de escaneo en dos dimensiones del lector óptico de una unidad de CD para implementarlas en un sistema de micro-espectroscopia de Rhaman miniaturizado. Lange et al. (2005) desarrollaron una forma de medir eventos vinculantes biomoleculares, usando un lector de CD y una tinción de plata. Kostner \& Vellekoop (2005; 2007) usaron el cabezal óptico de un lector de DVD para enfocar el láser en un chip microfluídico con una celda de flujo en citometría.

La intensidad de la señal óptica reflejada se veía influida por las partículas que atravesaba el haz y se medía directamente en el lector óptico. Más adelante probaron el sistema con microesferas de poliestireno (Kostner \& Vellekoop, 2008). Yim et al. (2008) desarrollaron un método para análisis de micro-estructuras. La fluorescencia emitida y la luz reflejada por dispositivos microfluídicos eran detectadas por un tubo foto-multiplicador para obtener alta sensibilidad de detección, mientras el cabezal de una unidad de DVD ofrecía la función de auto-enfoque, manteniendo una iluminación constante. Kim et al. $(2007$; 2008) propusieron un 
nuevo método de detección óptica para chips de $\mathrm{ADN}$ que utilizaba el cabezal óptico de una unidad de DVD y una capa reflectante que se adjuntaba a las láminas de chips de ADN. La luz reflejada por esta capa, medida con el cabezal óptico, se utilizaba para el auto-enfoque.

El sistema de auto-enfoque permitía que el rayo láser permaneciera enfocado el chip de ADN, excitando un colorante Cy5 que emitía una señal de fluorescencia. La intensidad de esta señal se medía en un detector. Park et al. (2008) desarrollaron un sistema de detección de bio-información a partir de un bio-chip conformado por un sustrato con una capa de grabación por cambio de fase, similar a la encontrada en un CD o DVD. El proceso de escritura de datos en un CD o DVD consiste en transferir un código en la superficie del disco, por medio de irradiación con un láser. El material del disco cambia su fase de estado cristalino a estado amorfo (y por tanto cambia su reflectividad) en las partes calentadas por el láser. Park et al., encontraron que cuando ocurre una hibridización de $\mathrm{ADN}$ por medio de sondas de $\mathrm{ADN}$ adjuntas a nanopartículas de metal, las nano partículas de metal alteran la transmitancia de la energía láser irradiada sobre la superficie del CD durante el proceso de transferencia de datos. La diferencia en la reflectividad resultante se puede leer en una unidad de CD/DVD.

\subsection{Otras aplicaciones}

Armstrong \& Fitzgerald (1992) desarrollaron un autocolimador para detectar pequeñas inclinaciones angulares de superficies reflectantes. En el autocolimador, el haz de luz de la unidad de CD se dirige hacia la superficie reflectante y el haz reflejado sigue aproximadamente el mismo camino que el haz inicial a través de la lente objetivo, sin embargo, un cambio en la orientación angular del objeto a medir resulta en un movimiento del haz de luz reflejado, que se mide sobre la superficie del detector de cuatro cuadrantes.

Quercioli et al. (1997) presentaron una aplicación en velocimetría de correlación óptica donde los tres haces del cabezal óptico de la unidad de CD (que se originan cuando el láser atraviesa la 
red de difracción) se enfocan en la superficie a medir, de manera que los tres puntos queden alineados a lo largo de la dirección del movimiento. Los haces reflejados inciden en un sistema de tres detectores y correlacionando las señales detectadas, se puede calcular el tiempo de retardo entre ellas y por lo tanto, la velocidad del objeto. Quercioli et al. (1998) presentaron un velocímetro Doppler de láser en el cual las franjas producidas al iluminar la rejilla de difracción con el diodo láser se proyectaban sobre la superficie en movimiento y se orientaban perpendicularmente a la dirección de desplazamiento. La intensidad de la luz dispersada, medida por los fotodetectores, se procesaba usando transformada de Fourier. El espectro de potencia mostraba un pico en la frecuencia fundamental, dando información sobre la velocidad del objeto.

Quercioli et al. (1999a) desarrollaron un interferómetro de Fizeau multifase homodino que hace uso de la detección de enfoque astigmática presente en una unidad de CD/DVD. El detector de cuadrante de la unidad ofrecía cuatro señales de fotocorriente cuyos retardos de fase podían controlarse fácilmente, permitiendo la aplicación de algoritmos de interferometría de desplazamiento de fase.

Fan et al. (2003) desarrollaron un sistema de medición de rectitud usando el cabezal óptico de una unidad de DVD, a la cual le quitaron la lente objetivo mientras una pieza con borde afilado se colocaba entre el cabezal y un espejo plano. La parte no sombreada del haz de láser se reflejaba y se enfocaba en el fotodetector de cuatro cuadrantes, cuyas señales se sumaban para medir la potencia del láser reflejado. El análisis teórico basado en la teoría de la distribución de Gauss circular permitía calcular el error de la rectitud del borde afilado mientras éste se movía.

En el área de fluidos también se han aplicado las unidades de CD. Fabricando numerosos canales en un CD, que irradian desde el centro del disco hacia el borde externo, se puede bombear un líquido utilizando la fuerza centrífuga que se genera cuando el CD gira en la unidad (Felton, 2003). La fuerza centrífuga de bombeo sólo depende de la viscosidad y la velocidad de rotación. La detección 
de drogas y uniones en células biológicas se han convertido en los primeros uso de estos desarrollos (Felton, 2003).

Fan et al. (2005) adoptaron el cabezal óptico de una unidad de DVD como sonda de perfil con precisión sub-micrométrica para una micro-CMM (Máquina de medición por coordenadas), usando el principio de error de enfoque de la unidad de DVD. Los movimientos de cada eje de la micro-CMM se conseguían por medio de motores lineales de piezo-cerámica (PCLM). Más adelante, Fan et al. (2007) propusieron un diseño modificado que usaba un interferómetro de rejilla de difracción lineal (LDGI) de mayor tolerancia, una nueva estructura de huso y un nuevo esquema de control de movimiento.

Morris et al. (2006) diseñaron un pulsador electrónico basado en una unidad de DVD para generar flujos de pulso en la simulación de sistemas de detección de rayos gamma y neutrones. Utilizando un computador, escribieron en un DVD más de una hora de información con espectros reales o simulados de rayos gamma y pulsos de neutrones. Este DVD lo introdujeron en una unidad de DVD extraída de un computador a la cual adjuntaron un panel. Chu \& Lin (2005) desarrollaron un acelerómetro óptico que dirigía el láser de una unidad de DVD sobre la masa sísmica y utilizaba la señal de error de enfoque (FES) detectada por el fotodiodo de cuatro cuadrantes para medir el desplazamiento de la masa sísmica respecto a la base del acelerómetro. Más adelante, Chu et al. (2007) desarrollaron un acelerómetro óptico bi-dimensional usando este mismo concepto.

Chu \& Chiu (2007) desarrollaron una sonda táctil de tres dimensiones y de bajo costo usando dos sensores basados en el cabezal óptico de una unidad de CD/DVD. El primer sensor medía el movimiento de traslación de la sonda en la dirección del eje $z$, mientras que el segundo medía las rotaciones angulares de la sonda sobre los ejes $x$ e $y$. El movimiento de traslación, se medía gracias a que un desplazamiento $\delta \mathrm{Az}$ producía un cambio de la forma del punto de luz enfocado sobre el fotodiodo de cuatro cuadrantes en la unidad de CD/DVD, mientras que los cambios angulares producían un cambio en la posición de dicho punto de luz. Utilizando este 
mismo principio, Hwu et al. (2007) construyeron un sistema para medir simultáneamente los desplazamientos verticales e inclinaciones angulares en dos dimensiones de elementos micromecanizados. Más adelante, Hwu et al. (2008) demostraron que el sistema podía lograr mediciones en tiempo real y aplicaron el sistema a un microscopio de fuerza atómica (AFM) para medir la desviación del voladizo microfabricado.

\section{Conclusiones}

De la revisión realizada se encontró que muchos de los avances logrados en las diferentes aplicaciones de las unidades de CD/DVD y los ratones de computador, se basaron en el desarrollo de nuevas tecnologías para estos dispositivos. El paso del ratón opto-mecánico al ratón óptico generó toda una serie de aplicaciones en robótica y el paso de la unidad de CD a la unidad de DVD permitió usar un láser enfocado con menor longitud de onda y mayor precisión. Siguiendo esta tendencia, se vislumbran nuevos desarrollos en las mismas áreas empleando la iluminación láser de los nuevos ratones ópticos y las unidades de blue ray, que probablemente llegarán a reemplazar a las de CD/DVD.

Muchas aplicaciones cuya principal ventaja podría ser el bajo costo se ven opacadas al usar elementos costosos, como motores o plataformas piezoeléctricas. En este sentido se destaca el trabajo de Islam et al. (2007) quienes desarrollaron un perfilómetro con una unidad de DVD y un ratón óptico para medir la posición del sensor, evitando el uso de plataformas piezo-eléctricas costosas. Nuevos desarrollos enmarcados en el bajo costo podrían lograrse siguiendo esta misma tendencia.

En casi todas las aplicaciones se aprovechan muy pocos elementos presentes en el dispositivo, especialmente en el caso de las unidades de CD/DVD donde se ha utilizado casi que exclusivamente la tecnología encontrada en la cabeza del lector óptico de la unidad. Estas unidades tienen además un motor de desplazamiento preciso para mover el cabezal, otro para hacer girar el CD/DVD y otro más 
para el movimiento de la carcasa dentro del computador, además de sistemas de reducción que usan engranajes, poleas, cremalleras y sinfín. Estos mismos elementos se podrían usar para realizar escaneos sobre superficies en microscopía o perfilometría, tal como hicieron Bartoli et al. (2001a; 2001b), quienes desarrollaron un perfilómetro unidimensional en el cual el escaneo de la muestra lo realizaba el motor de desplazamiento del cabezal óptico. El aprovechamiento de una mayor cantidad elementos permite lograr desarrollos de muy bajo costo y generar mejor impacto ambiental. En este sentido, futuras aplicaciones de la unidad de CD/DVD deberían buscar un mayor reaprovechamiento de los elementos de la unidad, pudiendo por ejemplo usarlos en el diseño de un mecanismo de movimiento en dos dimensiones para aplicaciones en perfilometría o microscopía.

La mayor aplicación que se ha dado a los ratones de computador es en el área de la robótica, sin embargo, la principal limitación de estos sensores se debe al hecho de que la distancia entre el ratón y la superficie es crítica para obtener medidas precisas y a que los ratones no pueden operar a altas velocidades. Solo dos trabajos se han ocupado directamente de este problema: el de Bradshaw et al. (2007) quienes añadieron la lente de una cámara CCD para ampliar el espacio proyectado sobre el sensor del ratón, superando las limitaciones de velocidad y distancia de la superficie; y el de Hyun et al. (2009), quienes diseñaron un sensor óptico diferencial que permitía medir desplazamientos independientemente de las variaciones en la altura del robot (dentro de un rango). Una integración de ambos trabajos podría resultar en un sistema mejorado respecto a velocidad del sensor, distancia desde la superficie y poca sensibilidad a variaciones de altura. También podría explorarse el uso del sensor diferencial de Hyun et al., en medición de vibraciones, ya que este no se ve afectado por cambios en la distancia.

Los ratones ópticos, siendo esencialmente cámaras de alta velocidad con un sistema de procesamiento de imágenes, podrían en teoría ser aplicados en muchos campos del reconocimiento de 
patrones en imágenes, tal como lo emplearon Tresanchez et al. para la detección de monedas falsas (Tresanchez et al., 2009a) y para desarrollar un codificador absoluto (Tresanchez et al., 2010). Siguiendo esta tendencia podría explorarse la aplicación del ratón óptico en otras áreas de reconocimiento de patrones, por ejemplo en un sistema de reconocimiento de huellas dactilares.

Finalmente, en las aplicaciones de la unidad de CD/DVD en el campo de microscopía se observa que la aplicación del dispositivo está limitada casi exclusivamente a la medición del desplazamiento de un voladizo o una sonda (en microscopía de fuerza atómica o en microscopía de sonda de barrido). Estas aplicaciones usan el mismo principio de auto-enfoque que usan las aplicaciones de perfilometría. Sin embargo, la cabeza óptica de una unidad de CD/DVD tiene todos los elementos para desarrollar un microscopio óptico, de manera que pueden lograrse otras aplicaciones microscopía óptica, en las que se podría además integrar una cámara de bajo costo para la adquisición digital de imágenes.

Con base en los resultados obtenidos en esta revisión, en el centro de investigación del INSTITUTO TECNOLÓGICO METROPOLITANO se está planteando un trabajo encaminado a desarrollar un microscopio óptico motorizado empleando reciclaje tecnológico de una unidad de CD/DVD, un ratón óptico y una cámara web. Adjuntado la cámara web a la cabeza óptica de la unidad, se lograría la adquisición de imágenes a través de un computador. Se pretende utilizar el movimiento del cabezal óptico para lograr desplazamientos en una dirección y diseñar un mecanismo de desplazamiento en la dirección perpendicular, usando los elementos presentes en la unidad. El ratón óptico se emplearía para medir los desplazamientos y realimentar el control de posición. De esta forma se tiene una aplicación que es de muy bajo costo y aprovecha al máximo las partes encontradas en estos dispositivos. De lograrse este desarrollo, podrían usarse las unidades de CD/DVD y los ratones ópticos de equipos desechados para construir microscopios de bajo costo para dotar los laboratorios de muchas escuelas públicas en Colombia. 


\section{Referencias}

Agilent Technologies, (2001); Optical mice and how they work: the optical mouse is a complete imaging system in a tiny package. White paper, Agilent Technologies, 4 p. Disponible en: https://electronics.wesrch.com/ User images/Pdf/1TZ 1193720648.pdf

Armstrong, T.R., Fitzgerald, M.P., (1992); An autocollimator based on the laser head of a compact disc player. Measurement Science and Technology, 3(11), 1072-1076.

Bartoli, A., Poggi, P., Quercioli, F., Tiribilli, B., (2001a); Fast one dimensional profilometer with a compact disc pickup. Applied Optics, 40(7), 1044-1048.

Bartoli, A., Poggi, P., Quercioli, F., Tiribilli, B., Vassalli, M., (2001b); Optical profilometer with a standalone scanning sensor head. Optical Engineering, 40(12), 2852-2859.

Benschop, J., Rosmalen, G., (1991); Confocal compact scanning optical microscope based on compact disc technology. Applied Optics, 30(10), 1179-1184.

Bonarini, A., Matteucci, M., Restelli, M., (2004); A kinematic-independent dead-reckoning sensor for indoor mobile robotics. En Proceedings of 2004 IEEE/RSJ International Conference on Intelligent Robots and Systems, 3750-3755.

Bonarini, A., Matteucci, M., Restelli, M., (2005); Automatic error detection and reduction for an odometric sensor based on two optical mice. En Proceedings of the 2005 IEEE International Conference on Robotics and Automation, 1675-1680.

Bradshaw, J., Lollini, C., Bishop, B., (2007); On the development of an enhanced optical mouse sensor for odometry and mobile robotics education. En 39th Southeastern Symposium on System Theory, 6-10.

Chu, C.L., Lin, C.H., (2005); Development of an optical accelerometer with a DVD pick-up head. Measurement Science and Technology, 16(12), 2498-2502.

Chu, C.L., Lin, C.H., Fan, K.C., (2007); Two-dimensional optical accelerometer based on commercial DVD pick-up head. Measurement Science and Technology, 18(1), 265-274.

Chu, C.L., Chiu, C.Y., (2007); Development of a low-cost nanoscale touch trigger probe based on two commercial DVD pick-up heads. Measurement Science and Technology, 18(7), 1831-1842. 
Cooney, J.A., Xu, W.L., Bright, G., (2004); Visual dead-reckoning for motion control of a Mecanum-wheeled mobile robot. Mechatronics 14(6), $623-637$.

Courreges, F., Edkie, A., Poisson, G., Vieyres, P., (2009); Ergonomic mouse based interface for 3D orientation control of a tele-sonography robot. En The 2009 IEEE/RSJ International Conference on Intelligent Robots and Systems, 61-66.

Doherty, J.A., Pires, A., (1987); A new microcomputer-based method for measuring walking phonotaxis in field crickets (Gryllidae); Journal of Experimental Biology, 130(1), 425-432.

Donatell, G., Meister, D., O’Brien, J., Thurlow, J., Webster, J., Fellow, L., Salvi, F., (2005); A simple device to monitor flexion and lateral bending of the lumbar spine. IEEE Transactions on Neural Systems and Rehabilitation Engineering, 13(1), 18-23.

Ehrmann, K., Ho, A., Schindhelm, K., (1998); A 3D optical profilometer using a compact disc reading head. Measurement Science and Technology, 9(8), 1259-1265.

Fan, K.C., Chu, C.L., Mou, J.I., (2001); Development of a low-cost autofocusing probe for profile measurement. Measurement Science and Technology, 12(12), 2137-2146.

Fan, K.C., Chu, C.L., Liao, J.L., Mou, J.I., (2003); Development of a highprecision straightness measuring system with DVD pick-up head. Measurement Science and Technology, 14(1), 47-54.

Fan, K.C., Fei, Y., Yu, X., (2005); Development of a micro-CMM. En Proceedings of the International Manufacturing Leaders Forum on "Global Competitive Manufacturing”, 27th February - 2nd March 2005, Adelaide, Australia. $7 \mathrm{p}$.

Fan, K.C., Lai, Z.F., Wu, P. Chen, Y.C., Chen, Y., Jäger G., (2007); A displacement spindle in a micro/nano level. Measurement Science and Technology, 18(6), 1710-1717.

Felton, M., (2003); CD simplicity. Analytical Chemistry, 75(13), 302A-306A.

Gintautas, V., Hübler, A., (2009); A simple, low-cost, data-logging pendulum built from a computer mouse. Physics Education 44 (5), 488-491.

Handler, J., Ochoa, O., Kolp, N., (1996); A mouse in our laboratory. The Physics Teacher, 34(8), 488-490.

Hu, J.S., Cheng, J. H., Chang, Y. J., (2007); Spatial trajectory tracking control of omni-directional wheeled robot using optical flow sensors. En 
16th IEEE International Conference on Control Applications, part of IEEE Multi-conference on Systems and Control, 1462-1467.

Hu, J., Chang, Y., Hsu, Y., (2009); Calibration and on-line data selection of multiple optical flow sensors for odometry applications. Sensors and Actuators, 149(1), 74-80.

Hwu, E.T., Huang, K.Y., Hung, S. ., Hwang, I.S., (2006); Measurement of cantilever displacement using a compact disk /digital versatile disk pickup head. Japanese Journal of Applied Physics, 45(3B), 2368-2371.

Hwu, E.T., Hung, S.K., Yang, C.W., Hwang, I.S., (2007); Simultaneous detection of translational and angular displacements of micromachined elements. Applied Physics Letters, 91(22), 221908-221908-3.

Hwu, E.T., Hung, S.K., Yang, C.W., Huang, K.Y., Hwang, I.S., (2008); Realtime detection of linear and angular displacements with a modified DVD optical head. Nanotechnology, 19(11), 115501-115507.

Hwu, E.T., Illers, H., Jusko L., Danzebrink, H.U., (2009); A hybrid scanning probe microscope (SPM) module based on a DVD optical head. Measurement Science and Technology, 20(8), 1-8.

Hyun, D., Yang, H.S., Park, H.R., Park, H.S., (2009); Differential optical navigation sensor for mobile robots. Sensors and Actuators A: Physical, 156(2) 296-301.

Islam, N., Parkin, R., Jackson, M., Mueller, P., (2007); A novel surface profile measurement system. AU Journal of Technology 10(3), 203-209.

Jackson, J., Callahan, D., Marstrander, J., (2007); A rationale for the use of optical mice chips for economic and accurate vehicle tracking. En IEEE Conference on Automations Science and Engineering, 939-944.

Jones, C.L., (2005); Cryptographic hash functions and CD-based optical biosensors. Problems of Nonlinear Analysis in Engineering Systems. 2(23), $17-36$.

Kim, J., Liu, G., Lee, L., (2005); Lens-scanning Raman microspectroscopy system using compact disc optical pickup technology. Optics Express, 13(12), 4780-4785.

Kim, S., Kim, H., (2006); Robust optical odometry using three optical mice. Journal of Control, Automation and Systems Engineering, 12(9), 861867.

Kim, K.H., Lee, S.Y., Kim, S., Lee, S.H., Jeong, S.G., (2007); A new DNA chip detection mechanism using optical pick-up actuators. Microsystem Technologies, 13(8-10), 1359-1369. 
Kim, S., Jeong, I., Lee, S., (2007); Mobile robot velocity estimation using an array of optical flow sensors. En International Conference on Control, Automation and Systems, Oct. 17-20, Seoul, Korea, 616-621.

Kim, K.H., Lee, S.Y., Kim, S., Jeong, S.G., (2008); DNA microarray scanner with a DVD pick-up head. Current Applied Physics 8(6), 687-69.

Kim, S., Lee, S., (2008); Optical mouse array position calibration for mobile robot velocity estimation. En IEEE Conference on Robotics, Automation and Mechatronics, Chengdu, 18 Nov, 1167-1172.

Kostner, S., Vellekoop, M., (2005); Particle and cell detection using a DVD pickup head. En Biennial Report of The Society for Micro- and Nanoelectronics 2005-2006. 401-404.

Kostner, S., Vellekoop, M., (2007); Low cost cytometer based on a DVD pickup head. En Eleventh International Conference on Miniaturized Systems for Chemistry and Life Sciences, 739-741.

Kostner, S., Vellekoop, M.J., (2008); Cell analysis in a microfluidic cytometer applying a DVD pickup head. Sensors and Actuators B 132(2), 512-517.

Kricka, L., (1998); Miniaturization of analytical systems. Clinical Chemistry 44(9), 2008-2014.

Lange, S. et al (2005); Measuring bimolecular binding events with a compact disc player device. Angewandte Chemie, 45(2), 270-273.

Lee, C.H., Chang, C.T., Fan, K.S., Chang, T.C., (2004); An overview of recycling and treatment of scrap computers. Journal of Hazardous Materials, 114(1-3), 93-100.

Lee, S., Song, J.B (2004a); Robust mobile robot localization using optical flow sensors and encoders. En Proceedings of the IEEE International Conference on Robotics and Automation, New Orleans, LA, April 2004, 1039-1044.

Lee, S., Song, J.B., (2004b); Mobile robot localization using optical mice. En Proceedings of the 2004 IEEE Conference on Robtics, Automation and Mechatronics, 1192-1197.

Lott, G., Rosen, M., Hoy, R., (2007); An inexpensive sub-millisecond system for walking measurements of small animals based on optical computer mouse technology. Journal of Neuroscience Methods, 161(1), 55-61.

Mansuripur, M., Sincerbox, G., (1997); Principles and techniques of optical data storage. Proceedings of the IEEE, 85(11), 1780-1796.

Marthaler, C., (2008); Computers for Schools: sustainability assessment of supply strategies in developing countries. A case study in Colombia. 
Master Thesis, Department of Environmental Science, Swiss Federal Institute of Technology Zürich (ETH), 127 p.

Mason, A.C., Oshinsky, M.L., Hoy, R.R., (2001); Hyperacute directional hearing in a microscale auditory system. Nature, 410(6829), 686-690.

Mei, T.C., (2005); Understanding optical mice, White-Paper, Avago Technologies, September 14, 8 p. Disponible en: www.avagotech.com

Minoni, U., Signorini, A., (2005); Low-cost optical motion sensors: An experimental characterization. Sensors and Actuators A: Physical, 128(2), $402-408$.

Morris, S., Pratt, R., Hughes, M., Kouzes, R., Pitts, K., Robinson, E. (2006); DVD based electronic pulser. IEEE Transactions on Nuclear Science, 53(4), 2303-2307.

Nakao, T., Kashitani, A., Kaneyoshi, A., (1998); Scanning a document with a small camera attached to a mouse. En Proceedings of the 4th IEEE Workshop on Applications of Computer Vision, 63-68.

Ng, T.W., (2003); The optical mouse as a two-dimensional displacement sensor. Sensors and Actuators A: Physical, 107(1), 21-25.

Ng, T.W., (2004); Measuring viscoelastic deformation with an optical mouse. Journal of Chemical Education, 81(11), 1628-1629.

Ng, T.W., Ang, K.T., (2004); The optical mouse for vibratory motion sensing. Sensors and Actuators A: Physical, 116(2), 205-208.

Ng, T.W., Cheong, T.L., (2004); The optical mouse as an inexpensive regionof-interest position recorder in optical microscopy. Microscopy Research and Technique, 63(4), 203-205.

Ng, T.W., Ang, K.T., (2005); The optical mouse for harmonic oscillator experimentation. American Journal of Physics, 73(8), 793-795.

Ng, T.W., Cheong, T.L., Sheridan, J., (2007); Digital readout manometer using an optical mouse. European Journal of Physics, 28(2), N11-N16.

Ochoa, O., Kolp, F., Handler, J., (1998); Quantitative demonstration of Lenz's law. The Physics Teacher, 36(1), 50-51.

Ott, D., (2008); Gestión de residuos electrónicos en Colombia: diagnóstico de computadores y teléfonos celulares. Reporte de EMPA y CNPMLTA, Medellín, Colombia, 146 p. Disponible en: http://ewasteguide.info/files/ Ott 2008 Empa-CNPMLTA.pdf 
Palacin, J., Valganon, I., Pernia, R., (2006); The optical mouse for indoor mobile robot odometry measurement. Sensors and Actuators A: Physical, 126(1), 141-147.

Park, K.H. et al (2008); Bio-information scanning technology using an optical pick-up head. Ultramicroscopy 108(10), 1319- 1324.

Puckett, J., Smith, T., (2002); Exporting harm: the high-tech trashing of Asia. The Basel Action Network. Seattle: Silicon Valley Toxics Coalition, August 14, 54 Disponible en: www.ban.org/E-waste/technotrashfinalcomp.pdf

Quercioli, F., Mannoni, A., Tiribilli, B., (1997); Correlation optical velocimetry with a compact disk pickup. Applied Optics, 36(25), 6372-6375.

Quercioli, F., Mannoni, A., Tiribilli, B., (1998); Laser Doppler velocimetry with a compact disc pickup. Applied Optics, 37(25), 5932-5937.

Quercioli, F., Tiribilli, B., Bartoli, A., (1999a); Interferometry with optical pickups. Optics Letters, 24(10), 670-672.

Quercioli, F., Tiribilli, B., Ascoli, C., Baschieri, P., Frediani, C., (1999b); Monitoring of an atomic force microscope cantilever with a compact disk pickup. Review of Scientific Instruments, 70(9), 3620-3624.

Sekimori, D., Miyazaki, F., (2005); Dead-reckoning for mobile robots using multiple optical mouse sensors. Transactions of the Society of Instrument and Control Engineers, 41(10), 775-782.

Sorensen, D.K., Smukala, V., Ovinis, M., Lee, S., (2003); On-line optical flow feedback for mobile robot localization/navigation. En Proceedings of the 2003 IEEE/RSJ international Conference on Intelligent Robots and Systems, $1246-1251$.

Tibbe, A., deGrooth, B., Greve, J., Rao, C., Dolan, G., Terstappen, L., (2002); Cell analysis system based on compact disk technology. Cytometry 47(3), 173-182.

Tresanchez, M., Pallejà, T., Teixidó, M., Palacín, J., (2009a); Using the optical mouse sensor as a two-euro counterfeit coin detector. Sensors, 9(9), pp. 7083-7096.

Tresanchez, M. Pallejà, T., Teixidó, M., Palacín, J., (2009b); The optical mouse sensor as an incremental rotary encoder. Sensors and Actuators A: Physical, 155(1), 73-81.

Tresanchez, M. Pallejà, T., Teixidó, M., Palacín, J., (2010); Using the image acquisition capabilities of the optical mouse sensor to build an absolute rotary encoder. Sensors and Actuators A: Physical, 157(1), 161-167. 
Tunwattana, N., Roskilly, A., Norman, R., (2008); Investigations into the effects of illumination and acceleration on optical mouse sensors as contact-free 2D measurement devices. Sensors and Actuators A: Physical, 149(1), 87-92.

Wang, X., Shida, K., (2008); Optical mouse sensor for detecting height variation and translation of a surface. En IEEE International Conference on Industrial Technology, Chengdu, 21-24 April, 1-6

Wang, X., Shida, K., (2009); Surface shape analyzing device using optical mouse sensor. En IEEE Youth Conference on Information, Computing and Telecommunication, Beijing, 20-21 September, 255-258.

Yang, Z., Maeda, R., (2000); Automatic micro flow rate measurement using a modified computer mouse device. En $1^{\text {st }}$ Annual International IEEEEMBS Special Topic Conference on Microtechnologies in Medicine \& Biology, 288-291.

Yim, V., Lee, S.Y., Kim, S., Park, J.Y., (2008); Multipurpose DVD pick-up scanner for analysis of microfluidics and micromechanical structures. En 30th Annual International IEEE EMBS Conference, 2749-2751.

Zhang, J.H., Cai, L., (1997a); An autofocusing measurement system with a piezoelectric translator. IEEE/ASME Transactions on Mechatronics, 2(3), 213-216.

Zhang, J.H., Cai, L., (1997b); Profilometry using an optical stylus with interferometric readout. Measurement Science \& Technology, 8(5), 546-549. 